\title{
Características clínico-epidemiológicas de los pacientes de la cohorte Lupus Paraguay (2013-2014)
}

\author{
*Marcos Aurelio Vázquez, Elías Rojas, Jonathan Losanto, Karin Bauman, María Eugenia Acosta, Gabriela \\ Avila, Marcia Melo, María Margarita Duarte, *Isabel Acosta Colman \\ ${ }^{1}$ Universidad Nacional de Asunción, Hospital de Clínicas, Servicio de Reumatología. San Lorenzo, Paraguay
}

Cómo referenciar este artículo/ How to reference this article:

\author{
Vázquez MA, Rojas E, Losanto J, Bauman K, \\ Acosta ME, Avila G, et al. Características clínico- \\ epidemiológicas de los pacientes de la cohorte Lupus \\ Paraguay (2013-2014). Mem. Inst. Investig. Cienc. \\ Salud. 2019; 17(1): 69-74
}

\section{R E S U M E N}

El lupus eritematoso sistémico (LES) es una enfermedad autoinmune, de etiología desconocida que presenta manifestaciones clínicas de gravedad y curso impredecible. Se ha observado en varias cohortes que esta enfermedad tiene un comportamiento más agresivo en la población no caucásica debido a diferencias socioeconómicas, demográficas y genéticas. El objetivo del estudio es describir las características clínico-epidemiológicas de los pacientes con LES incluidos en la cohorte LUPUS-PY y la situación actual de las muestras custodiadas en el BIOBANCO IMID-PY. Estudio basado en la revisión de historias clínicas y en los cuestionarios clínico-epidemiológicos del día de la inclusión al registro LUPUS-PY. Se calcularon las frecuencias para las variables categóricas y las medias con su desvío estándar para las continuas. Se incluyeron de forma consecutiva, 83 pacientes con diagnóstico de LES (edad media: $34,4 \pm 10,0$ años), el $86,7 \%$ era del sexo femenino, el $55 \%$ provenía de Asunción, el 49,1\% tenía nivel educativo básico, el $45 \%$ percibía un ingreso mensual de un sueldo mínimo (aproximadamente 350 dólares americanos) o menos. El 46,8\% no realizaba ningún tipo de actividad física. La media del tiempo trascurrido desde el inicio de los síntomas y el diagnóstico del LES fue de 9,3 $\pm 13,7$ años y el tiempo de evolución de la

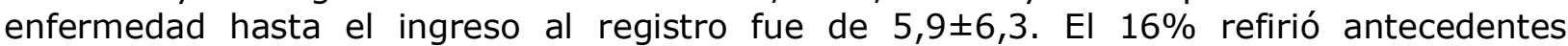
familiares de enfermedades autoinmunes. La primera manifestación de la enfermedad más descripta fue la articular $(45 \%)$. Los pacientes con LES de nuestro centro tienen características tanto clínicas como epidemiológicas similares a las reportadas por otras cohortes.

Palabras claves: lupus eritematoso sistémico, Registro LUPUS-PY, características clínicoepidemiológicas.

\section{Characteristics clinical-epidemiological of the patients of the Lupus Paraguay cohort (2013-2014)}

\footnotetext{
A B S T R A C T

Systemic lupus erythematosus (SLE) is an autoimmune disease of unknown etiology that presents severe clinical manifestations and unpredictable course. It has been observed in several cohorts that this disease has a more aggressive behavior in the non-Caucasian population due to socioeconomic, demographic and genetic differences. The objective of this study was to describe the clinical-epidemiological characteristics of SLE patients included in the LUPUS-PY cohort and the current situation of the samples stored in the BIOBANCO IMID-PY. The study was based on the review of clinical histories and clinical-epidemiological questionnaires on the day of inclusion in the LUPUS-PY registry. Frequencies were calculated for the categorical variables and the means with their standard deviation for the

Fecha de recepción: noviembre 2018. Fecha de aceptación: febrero 2019

*Autor Correspondiente: Dra. Isabel Acosta. Universidad Nacional de Asunción, Hospital de Clínicas, Servicio de Reumatología. San Lorenzo Paraguay

Email: dr.acostacolman@gmail.com
} 
continuous ones. A total of 83 patients with a diagnosis of SLE were included consecutively (mean age: $34.4 \pm 10.0$ years), $86.7 \%$ were female, $55 \%$ were from Asunción, $49.1 \%$ had basic education level, $45 \%$ received monthly a minimum salary (approximately 350 US dollars) or less. Forty six point eight percent did not perform any type of physical activity. The mean time elapsed since the onset of symptoms and the diagnosis of SLE was $9.3 \pm$ 13.7 years, and the time of evolution of the disease until entry into the registry was $5.9 \pm$ 6.3. Sixteen percent reported a family history of autoimmune diseases. The first manifestation of the disease more described was articular. Patients with SLE in our center have both clinical and epidemiological characteristics similar to those reported by other cohorts.

Keywords: systemic lupus erythematosus, LUPUS-PY registry, clinical and epidemiological characteristics.

\section{INTRODUCCIÓN}

El Lupus Eritematoso Sistémico (LES) es una patología crónica y multisistémica de etiología desconocida, definida por sus características clínicas y por la presencia de autoanticuerpos dirigidos contra uno o más componentes del núcleo ${ }^{(1-3)}$. El factor genético por sí solo no explica la etiología de la enfermedad. A esto se suman otros factores tales como los ambientales los cuales pueden participar en el desarrollo del LES en un huésped genéticamente susceptible La severidad en el lupus eritematoso sistémico puede estar influenciada por ciertos factores socioeconómicos, demográficos y genéticos ${ }^{(1,2,4)}$.

En el presente trabajo realizamos un análisis descriptivo de las características clínicas y epidemiológicas de los primeros pacientes incluidos en la Cohorte LUPUS-PARAGUAY (LUPUS-PY) con el objetivo de identificar las características epidemiológicas y clínicas de los pacientes paraguayos que padecen LES atendidos en el Departamento de Reumatología del Hospital de Clínicas en los años 2013-2014.

Dicha Cohorte dio sus primeros pasos a inicios del 2013, gracias al apoyo del Rectorado de la UNA, y constituye actualmente el primer registro asociado a un BIOBANCO de muestras biológicas de pacientes con diagnóstico de LES de origen paraguayo.

\section{MATERIALES Y MÉTODOS}

Estudio observacional que describe los datos registrados en los cuestionarios clínicosepidemiológicos de todos los pacientes, el día de la inclusión a la Cohorte LUPUS-PY. Cada paciente previa inclusión realizó la firma del consentimiento informado.

Para el análisis estadístico, se utilizó para las variables categóricas las frecuencias y porcentajes y para las continuas las medias con su desvío estándar respectivo. Para el análisis de esta cohorte se utilizó el paquete estadístico Epi Info versión 3.5.4.

\section{RESULTADOS}

Fueron incluidos de forma consecutiva 83 pacientes con diagnóstico de LES, el $13 \%$ $(11 / 83)$ de los pacientes pertenecían al género masculino y el $86,7 \%(72 / 83)$ de los pacientes al género femenino, con una relación hombre mujer $1: 7$. El 48\% (40/83) de los pacientes refería estar en pareja. En relación a las variables epidemiológicas, el 55\% (45/83) de los pacientes provenían de Asunción y el resto de gran Asunción y otras ciudades del interior del país (Tabla 1 ).

Tabla 1. Distribución de pacientes con LES según sexo, estado civil, procedencia. $N=83$

\begin{tabular}{lll}
\hline & Característica & Frecuencia (\%)(N) \\
\hline Género & Masculino & $13,3 \%(11 / 83)$ \\
& Femenino & $86,7 \%(72 / 83)$ \\
Estado Civil & En pareja & $48 \%(40 / 83)$ \\
& Sin parejas & $52 \%(43 / 83)$ \\
\multirow{2}{*}{ Procedencia } & Asunción & $55 \%(45 / 83)$ \\
& Gran asunción y otros & $45 \%(38 / 83)$ \\
Zona & Urbana & $67,5 \%(56 / 83)$ \\
\hline
\end{tabular}




\section{Rural}

$32,5 \%(27 / 83)$

El valor de la media del número de hijos por paciente fue de $1,3 \pm 1,6$ hijos. La media de la edad del ingreso al registro fue de 34,39 $\pm 10,03$ años. El $(55 / 83)$ de los pacientes contaba con nivel de educación básico. El 45\% (37/83) de los pacientes percibía un ingreso mensual menor o igual al sueldo mínimo (equivalente a 350 dólares americanos). El 42,3\% (51/83) de los pacientes convive con más de 3 habitantes en el domicilio y el $61,4 \%$ (51/83) refirió habitar en una casa perteneciente a la familia. El 67,5\% (56/83) procedía de una zona urbana. La profesión más frecuentemente registrada fue la de quehaceres domésticos. Tabla 2

Tabla 2. Distribución de pacientes con LES según nivel de educación, convivencia, propiedad e ingreso mensual. $\mathrm{N}=83$

\begin{tabular}{lll}
\hline \multicolumn{1}{c}{ Característica } & & Frecuencia (\%)(N) \\
\hline Propiedad & Propia (casa familiar)Alquiler & $61,4 \%(51 / 83)$ \\
& & $38,5 \%(32 / 83)$ \\
Convivencia & Más de 3 habitantes en una casa & $42,3 \%(51 / 83)$ \\
Nivel de educación & Básico & $66,26 \%(55 / 83)$ \\
& Superior & $33,7 \%(28 / 83)$ \\
Profesión & Quehaceres domésticos & $25 \%(21 / 83)$ \\
Ingreso Mensual & $\leq$ al sueldo mínimo & $45 \%(37 / 83)$ \\
\hline
\end{tabular}

El 70\% (58/83) de los pacientes refirió tener acceso a una asistencia sanitaria pública exclusivamente. Con respecto a los hábitos dietéticos, se objetivó un consumo medio semanal de leche de $609,2 \pm 410,7 \mathrm{ml}$, el consumo de otros alimentos se observan en la Tabla 3.

Tabla 3. Consumo de alimentos de los pacientes incluidos en la Cohorte LUPUS-PY.N=83

\begin{tabular}{ll}
\hline Alimentos encuestados & Cantidad (Media \pm DE) \\
\hline Leche (ml/día) & $609,2 \pm 410,7$ \\
Carne (porciones/semana) & $3,6 \pm 2,0$ \\
Huevo (número/semana) & $1,4 \pm 1,5$ \\
Frutas (porciones/semana) & $5,8 \pm 2,7$ \\
Verdura (porciones/semana) & $6,7 \pm 1,0$ \\
\hline
\end{tabular}

El 18\%(11/61) de los pacientes refirió el hábito tabáquico al momento de su inclusión en el registro. En relación a la actividad física de los pacientes, se objetivó que el 46,8\% (37/79) de los pacientes no realizaba ningún tipo de actividad física, tal y como se observa en la Figura 1. 


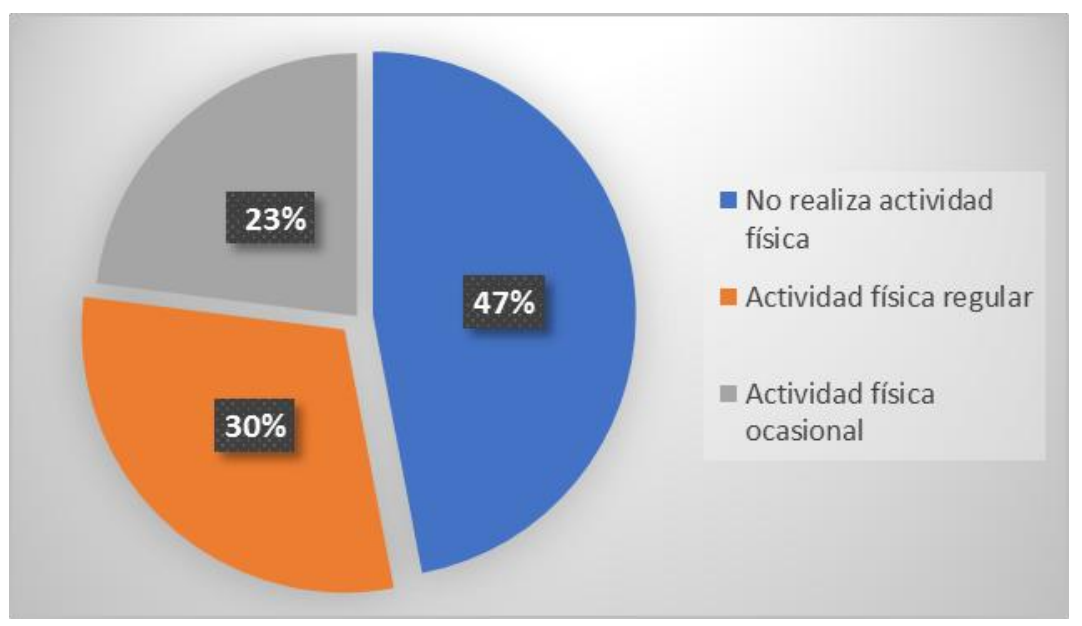

Figura 1. Distribución de pacientes con LES según la práctica de actividad física. $N=83$

En relación a las variables clínicas, en la Tabla 4 se observan los datos relacionados con (i.e. tiempo del inicio de los síntomas) y la edad (i.e. edad del diagnóstico del LES) de los pacientes incluidos en la cohorte.

Tabla 4. Características clínicas de los pacientes con LES. $\mathrm{N}=83$

\begin{tabular}{ll}
\hline \multicolumn{1}{c}{ Variable } & Media $\pm \mathbf{D E}$ \\
\hline $\begin{array}{l}\text { Tiempo desde el inicio de los síntomas al } \\
\text { diagnóstico }\end{array}$ & $9,27 \pm 13,72$ años \\
$\begin{array}{l}\text { Edad del diagnóstico de LES } \\
\text { Tiempo de evolución de la enfermedad hasta } \\
\text { el ingreso al registro }\end{array}$ & $5,9 \pm 6,29$ años \\
Edad del ingreso al registro & $34,39 \pm 10,03$ años \\
\hline
\end{tabular}

El 16\% (13/83) de los pacientes refirió antecedentes de una enfermedad autoinmune en la familia. La primera manifestación más frecuente fue la articular en un $45 \%(37 / 83)$ seguida por la afectación cutánea tal y como se observa en la Figura 2 . Al diagnóstico del LES el 39\% (32/83) de los pacientes presentó un ingreso hospitalario con una media de días de internación de $21,30 \pm 25,36$ días. El $30 \%$ de los pacientes presentó una complicación nefrológica durante su evolución hasta la fecha del ingreso.

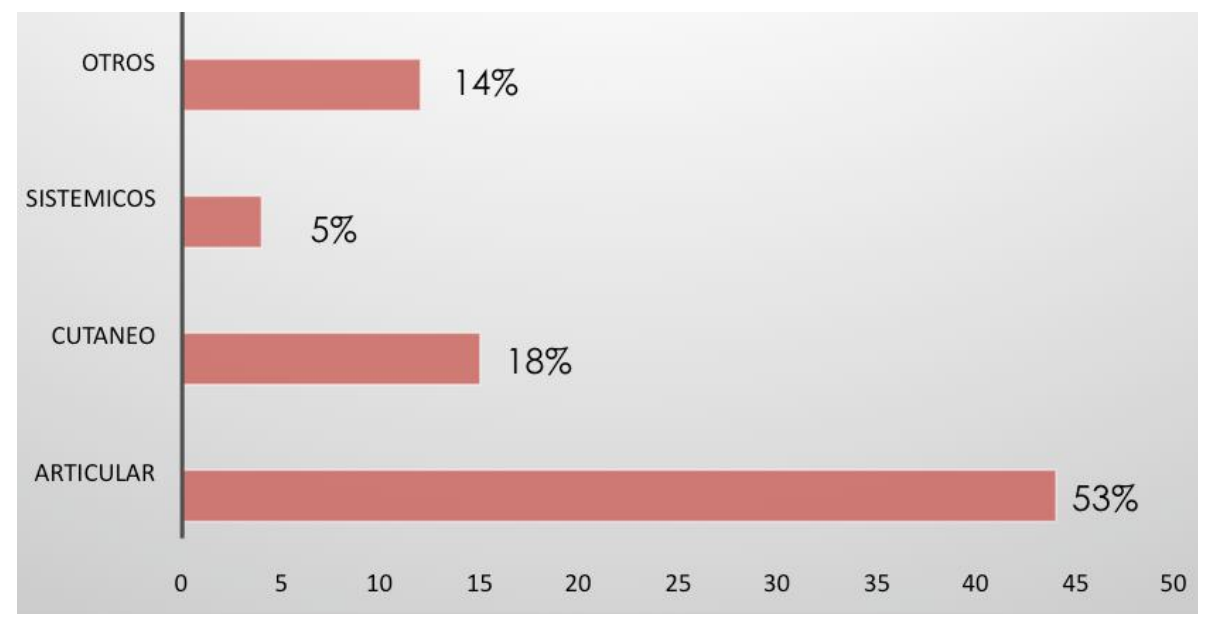

Figura 2. Manifestaciones al inicio de la enfermedad en pacientes con Lupus. $N=83$ 
El valor medio del SLEDAI (Systemic Lupus Erythematosus Disease Activity Index) del ingreso al registro fue de 2,45 $\pm 3,46$. El valor de la media del componente físico y mental del cuestionario SF36 fue de $42,2 \pm 12,6$ y $47,1 \pm 12,5$ respectivamente. Actualmente en el Biobanco se encuentran custodiadas aproximadamente 400 muestras de suero y 300 muestras de ADN codificadas (entre 3 a $10 \mu \mathrm{g}$ de ADN por paciente) procedentes de los 83 pacientes incluidos.

\section{DISCUSIÓN}

En este estudio se presentan las principales características de los primeros pacientes incluidos en la cohorte LUPUS-PY del Departamento de Reumatología del Hospital de Clínicas. Se observó un predominio del sexo femenino, coincidiendo con otras cohortes como la de LUMINA (Lupus in Minorities: Nature versus Nurture) y la de GLADEL (Grupo Latinoamericano de estudio de Lupus), cohortes importantes tanto europea como latinoamericana donde prima la heterogeneidad étnica y genética que confiere una mayor susceptibilidad y / o protección contra la enfermedad ${ }^{(5-8)}$.

El nivel educativo de nuestros pacientes fue predominantemente un nivel educativo básico coincidiendo con lo publicado por un estudio colombiano de Ariza et al, donde un $61,6 \%$ de los pacientes analizados tenían estudios de educación básica (i.e. primaria y secundaria) y según este estudio dicha situación puede influir en el pronóstico de la enfermedad $^{(9)}$. En este estudio colombiano también se observó que la ocupación, la edad avanzada, la poca educación y la baja condición económica se asociaban a una mayor actividad de la enfermedad en pacientes con LES $^{10,11}$. En nuestro estudio no analizamos esta asociación pero constatamos que en su mayoría nuestros pacientes refirieron pertenecer a familias numerosas y presentar ingresos económicos por debajo del salario mínimo estipulado en nuestro país.

Otro dato importante de recalcar es que la profesión más frecuentemente registrada en nuestro estudio fue la de quehaceres domésticos, coincidiendo con otros estudios como el estudio publicado por Ariza et al, quienes encontraron que un $42,3 \%$ eran amas de casa y en un $9,0 \%$ estaban desempleados, situación que refleja la condición laboral y por ende la dificultad de acceso a la atención sanitaria(12).

En relación a la actividad física de los pacientes, se objetivó que en su mayoría no realizaban actividad física. En otro estudio se observó que los pacientes obesos con LES eran más propensos a presentar una peor capacidad funcional, más altos niveles de fatiga y peor dolor, además de tener mayores concentraciones de mediadores de inflamación, tales como la proteína C reactiva (PCR) y la interleucina-6 (IL-6); que se encuentran más elevadas en pacientes con obesidad o sobrepeso que padecen de LES $\left({ }^{13-15)}\right.$.

Otra variable analizada en nuestra cohorte fue el hábito tabáquico, el cual no fue frecuente entre nuestros pacientes. No obstante, es importante recalcar la importancia del estudio y de la educación de nuestros pacientes sobre este hábito que está bien demostrado que se asocia con la aparición de más eventos cardiovasculares y peor pronóstico en este grupo de pacientes ${ }^{(16,17)}$.

En relación a las manifestaciones clínicas de nuestros pacientes, la manifestación inicial más frecuente fue la articular seguida por la afectación cutánea. La manifestación articular es también la afectación inicial más frecuente en otros estudios publicados ${ }^{(18,19)}$. En relación al compromiso renal, se pudo observar una alta prevalencia de esta complicación en nuestra cohorte, coincidiendo con otras cohortes de diferentes etnias ${ }^{(18,19)}$.También coincide con otras cohortes la edad media de diagnóstico del LES en nuestros pacientes, predominado en la edad fértil y más productiva de la vida laboral ${ }^{(18,19)}$.

A medida que se profundice el análisis de las diferentes cohortes existentes en el mundo, se logrará determinar en qué forma las características socioeconómicas y clínicas serán susceptibles de intervención y puedan influir en el pronóstico de los pacientes con LES en el tiempo ${ }^{(18,19)}$. Es por eso que estudios como este pueden contribuir con el conocimiento de la fisiopatología de esta enfermedad y de los posibles biomarcadores que determinan su curso.

\section{CONCLUSIÓN}

Los pacientes con LES de nuestro centro tienen características particulares tanto clínicas y epidemiológicas en relación a otras series de pacientes. Este reporte describe las características principales de los pacientes con LES incluidos hasta diciembre del 2014 en el Registro LUPUS-PY. 


\section{REFERENCIAS BIBLIOGRÁFICAS}

1. D'Cruz DP, Khamashta MA, Hughes GRV. Systemic lupus erythematosus Lancet 2007; 369: 587-96.

2. González LA, Toloza G SM, McGwin Jr. Alarcón GS. Ethnicity in systemic lupus erythematosus (SLE): Its influence on susceptibility and outcomes Lupus 2013; 22 (12): 214-24.

3. Fessel WF. Epidemiology of systemic lupus erythematosus. Rheum Dis Clin North Am $1988 ; 14: 15-23$

4. Hess EV. Role of drugs and environmental agents in lupus syndromes. Curr Opin Rheumatol 1992;4:688-692.

5. Atisha-Fregoso $Y$, Jakez-Ocampo J, Llorente L. Systemic lupus erythematosus in Hispanics. Autoimmunity. noviembre de 2011;44(7):555-61

6. Hahn BH, McMahon A MA, Wilkinson WD, Wallace DI, Daikh JD. Fitzgerald D. American College of Rheumatology guidelines for screening, treatment, and management of lupus nephritis Arthritis Care Res (Hoboken) 2012, 64: 797-808

7. Severiche Maury DM, Restrepo Escobar M, González Naranjo LA, Vanegas García AL, Muñoz Vahos $\mathrm{CH}$, Vásquez Duque $\mathrm{GM}$. (2014). A series of 115 patients with systemic lupus erythematosus: Clinical and immunological characteristics. Revista Colombiana de Reumatología 2014, 21(4), 183-192.

8. Pons-Estel BA, Catoggio LJ, Cardiel $\mathrm{MH}$, Soriano ER, Gentiletti S, Villa AR, et al. The GLADEL multinational Latin American prospective inception cohort of 1214 patients with systemic lupus erythematosus: Ethnic and disease heterogeneity among "Hispanics". Medicine 2004;83:1-17.

9. Oeser A, Chung CP, Asanuma Y, Avalos I, Stein CM. Obesity is an independent contributor to functional capacity and inflammation in systemic lupus erythematosus. Arthritis \& Rheumatism, 2005, 52(11), 3651-59.

10. Ariza K, Isaza P, Gaviria AM, Quiceno JM, Vinaccia S, Alvarán L, Márquez JD.. Calidad de vida relacionada con la salud, factores psicológicos y fisiopatológicos en pacientes con diagnóstico de Lupus Eritematoso Sistémico-LES. Terapia psicológica 2010, 28(1), 27-36.

11. Kulczycka L, Sysa-Jedrzejowska A, Zalewska-Janowska A, Miniszewska J, Robak E. Quality of life and socioeconomic factors in Polish patients with systemic lupus erythematosus. Journal of the European Academy of Dermatology and Venereology 2008; 22(10): 1218-26.

12. Chung CP, Avalos I, Oeser A, Gebretsadik, T, Shintani A, Raggi P, Stein CM. (2007). High prevalence of the metabolic syndrome in patients with systemic lupus erythematosus: association with disease characteristics and cardiovascular risk factors. Annals of the rheumatic diseases 2007; 66(2): 208-14.

13. Moss KE, Ioannou Y,Sultan SM, Haq I, Isenberg DA. Outcome of a cohort of 300 patients with systemic lupus erythematosus attending a dedicated clinic for over two decades Ann Rheum Dis 2002; 61 (5): 409-13

14. Alarcón GS, Friedman AW, Straaton KV, Moulds JM, Lisse J, Bastian HM, et al. Systemic lupus erythematosus in three ethnic groups: III. A comparison of characteristics early in the natural history of the LUMINA cohort. Lupus in Minority populations: Nature vs Nurture. Lupus. 1999;8:197-209.

15. Alarcón GS, McGwin G, Petri M, Reveille JD, Ramsey-Goldman R, Kimberly RP. Baseline characteristics of a multiethnic lupus cohort: PROFILE. Lupus 2002; 11: 95-101.

16. Toloza SMA, Uribe AG, McGwin G, Alarcón GS, Fessler BJ, Bastian HM, et al.Systemic lupus erythematosus in a multiethnic US cohort (LUMINA): XXII. Predictors of time to the occurrence of initial damage Arthritis Rheum 2004; 50: 3177-86

17. Petri M. Hopkins Lupus Cohort. 1999 update Rheum Dis Clin North Am 2000;26 (2): 199-213

18. Petri $M$, Purvey $S$, Fang $H$, Magder LS. Predictors of organ damage in systemic lupus erythematosus: the Hopkins Lupus Cohort. Arthritis \& Rheumatism 2012; 64(12), 4021-28

19. Alarcón GS, Roseman J, Bartolucci AA, Friedman AW, Moulds JM, Goel N et al. Systemic lupus erythematosus in three ethnic groups: Features predictive of disease activity early in its course. LUMINA Study Group. Lupus in minority populations, nature versus nurture. Arthritis \& Rheumatism 1998; 41 (7): 1173-80. 